\title{
Does Level of Engagement in a Digital Parent Training Program Impact Improvements in Parenting and Child Outcomes?
}

\author{
Jenna Brager ${ }^{1,2,3^{*}}$, BSN, RN, MS, PhD; Susan Breitenstein ${ }^{2 *}$, FAAN, PhD, BSN-RN; Chakra Budhathoki ${ }^{3^{*}}$, PhD;
} Deborah Gross ${ }^{3 *}$, FAAN, RN, DSC

\footnotetext{
${ }^{1}$ LifeBridge Health-Sinai Hospital of Baltimore, Baltimore, MD, United States

${ }^{2}$ College of Nursing, Ohio State University, Columbus, OH, United States

${ }^{3}$ School of Nursing, Johns Hopkins University, Baltimore, MD, United States

*all authors contributed equally
}

\section{Corresponding Author:}

Jenna Brager, BSN, RN, MS, PhD

LifeBridge Health-Sinai Hospital of Baltimore

$2401 \mathrm{~W}$ Belvedere Ave

Baltimore, MD

United States

Phone: 4109554766

Email: jbrager@lifebridgehealh.org

\begin{abstract}
Background: Approximately $8 \%$ to $10 \%$ of children younger than 5 years of age experience emotional, behavioral, and social relationship problems. These children are more likely to exhibit poor social interactions, problematic parent-child relationships, and school related setbacks, thus reinforcing the need for early interventions such as parent training programs. The ezParent program is a tablet-based delivery adaptation of the group-based Chicago Parent Program, a program designed to address the needs of families raising young children in urban poverty. The growing interest in and adoption of mHealth has changed the way people receive and seek treatment and the way clinicians deliver care. Despite the usefulness of mHealth apps in helping people manage various aspects of health, people's use of those technologies often lasts only for a short period of time. This suggests a need to delve more deeply into user behaviors.
\end{abstract}

Objective: The purpose of this study was to (1) classify levels of engagement by identifying individual usage of ezParent based on observed user activity (ie, "metadata") and (2) examine whether levels of ezParent engagement is associated with changes in parenting and child behavior over time (ie, parenting stress, self-efficacy, warmth, follow through, punishment, child behavior problems and intensity).

Methods: This study used a single-group, pre- and posttest design with repeated measures follow-up. Survey measures were collected at baseline (T1), 12 weeks postbaseline (T2) and 24 weeks postbaseline (T3). The study included 92 parents with data collected from two pediatric primary care clinics based in two urban cities with a high proportion of low income and minority families: Chicago, Illinois (cohort 1) and Baltimore, Maryland (cohort 2). Engagement was conceptualized based on total number of modules completed, amount of time spent in the program, and number of skills saved by the parent. Each outcome variable was modeled using a separate mixed-effects model to determine the model of best fit and was analyzed across time and level of engagement.

Results: Overall, 78 parents logged in to the ezParent program. The data aggregation resulted in 41 parents categorized as high engagers (cohort $1 \mathrm{n}=29$; cohort $2 \mathrm{n}=12$ ) and 37 parents as low engagers (cohort $1 \mathrm{n}=13$; cohort $2 \mathrm{n}=24$ ). Significant differences were across all outcome variables: parenting stress $(P<.05)$, self-efficacy $(P<.05)$, warmth $(P<.05)$, punishment $(P<.05)$, follow-through $(P<.05)$, child behavior intensity $(P<.05)$, and child behavior problems $(P<.05)$. Although parenting outcomes improved, improvements were not significantly associated with levels of engagement.

Conclusions: This study provides insight into engagement of parents participating in a digitally delivered parent training program. Although level of engagement was not associated with improvements in parenting and child outcomes, we were able to systematically identify and test key usage metrics to ope rationalize engagement. This indicates that further study may help researchers identify other usage metrics more indicative of engagement. By exploring usage data, researchers, app developers, and clinicians can better understand how users engage with future tablet-based interventions. 
(iproc 2019;5(1):e15104) doi: $10.2196 / 15104$

\section{KEYWORDS}

engagement; metadata; mobile health (mHealth); parent-child relations; child development; mobile apps

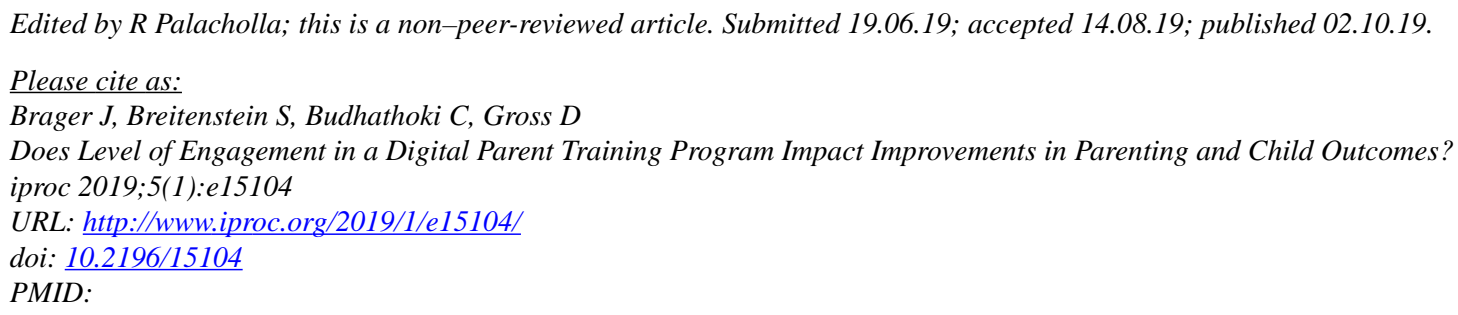

CJenna Brager, Susan Breitenstein, Chakra Budhathoki, Deborah Gross. Originally published in Iproceedings (http://www.iproc.org), 02.10.2019 This is an open-access article distributed under the terms of the Creative Commons Attribution License (https://creativecommons.org/licenses/by/4.0/), which permits unrestricted use, distribution, and reproduction in any medium, provided the original work, first published in Iproceedings, is properly cited. The complete bibliographic information, a link to the original publication on http://www.iproc.org/, as well as this copyright and license information must be included. 